See discussions, stats, and author profiles for this publication at: https://www.researchgate.net/publication/291328552

\title{
Convolution based Face Recognition using DWT and Feature Vector Compression
}

Conference Paper · December 2015

DOI: 10.1109/ICIIP.2015.7414814

4 authors, including:

Raja K B

University Visvesvaraya College of Engineering

188 PUBLICATIONS 1,340 CITATIONS

SEE PROFILE

Some of the authors of this publication are also working on these related projects:

Project Translation Based Face Recognition Using Fusion of LL and SV Coefficients View project

Project Face recognition View project 


\section{Convolution based Face Recognition using DWT and Feature Vector Compression}

\author{
Ganapathi V Sagar ${ }^{1}$, Savita Y Barker ${ }^{2}$ \\ ${ }^{1}$ Dr.Ambedkar Institue of Technology, \\ ${ }^{2}$ Sai Tektronix Pvt Ltd, \\ Bengaluru, India \\ ganapathi_sagar@yahoo.co.in
}

\author{
K B Raja ${ }^{3}, \mathrm{~K}$ Suresh Babu ${ }^{3}$ and Venugopal $\mathrm{K} \mathrm{R}^{3}$ \\ ${ }^{3}$ UniversityVisvesvaraya College of Engineering, \\ Bengaluru University \\ Bengaluru, \\ India
}

\begin{abstract}
Face Recognition is important Biometric credentials for identification or verification of a person. In this paper, we propose a novel technique of generating compressed unique features of face images which helps in improving matching speed of recognition. The training face database samples are applied to 2D-DWT to obtain LL band features. The LL band features are subjected to normalization to scale the magnitude values in the range 0 to 1 . The output of normalization is further convolved with the original face sample to obtain unique features. The convolved output is subjected to Gaussian filter to obtain smoothened image features. Further, The feature vector of several image samples of single person are compressed to convert into single vector to database feature vectors are created by compressing feature vectors of single person face samples in to single column unique vectors which helps in scaling down of feature vectors and improve matching speed. The test samples are subjected to same process to generate unique compressed test feature vectors and are compared with database vectors using Euclidean distance. The results are tabulated for different set of face databases and also compared with existing techniques to validate the performance of proposed method.
\end{abstract}

Keywords-Biometrics, face recognition, DWT, convolution, vector compression

\section{INTRODUCTION}

The physiological and behavioral characteristics of a person are used to recognize a living person which leads to Biometric system. The physiological traits are almost constant throughout the life time of a person and are fingerprint, palm print, Iris, DNA etc. The behavioral traits are varying based on mood, circumstances and environment around a person and examples are voice, signature, gait, keystroke etc. The biometric systems are broadly classified into verification system and identification system. In verifications system, the person's identity is declared by comparing features of a person with earlier stored features in the system which is one to one matching, i.e., the claim is accepted or rejected based on preconditions. In identification system, the person's identity is declared by comparing features of a person with earlier stored features of many persons i.e., one to many matching. The identification system is computationally expensive and complex, whereas, the verification system is simple, less expensive and complex.
The general biometric system has enrollment section, test section and matching sections. The biometric traits of persons are captured using sensors. The captured images are preprocessed and features are extracted to create database in the enrollment section. The biometric trait to be tested is captured using sensor, preprocessed and features are extracted to create single feature vector or matrix in the test section. The matching section is used to compare test biometric trait features with database biometric trait features to identify a person based on distance formulae or classifiers. The advantages of biometric identification system compared to traditional methods such as personnel identification number (PIN), identity card, smart card etc., are the biometric traits can't be lost as traits are attached to a person. The face recognition is used in online image search, surveillance in crowd, identification of terrorist in a mob, entry into corporate offices etc. The challenges in face recognition are variations in the light intensity, pose variations, hair occlusions, blinked eye etc.

\section{Contributions and novel aspects of this paper:}

The proposed method involves face recognition by using convolution, DWT and vector compression. The face database images are convolved with the output of normalized block obtained by applying 2D-DWT on face database images. The Gaussian filters helps in smoothening of high frequency edges after convolution. The filter outputs are many feature vectors based on number of face images per person and many feature vectors of a single person are converted into single feature vector by arithmetic addition. The test image features are compared with database image features using Euclidian distance.

\section{Organization:}

This paper is organized as follows: Section 1 gives brief introduction to Biometrics. Section 2 briefly reviews the literature; Section 3 introduces the new face recognition system based on convolution and compression of database vector features, section 4 presents the performance results and compares proposed method with existing methods. Section 5 concludes our work with future directions. 


\section{LITERATURE SURVEY}

In this section, the existing techniques of face recognition are discussed.

Jianwn wan et al., [1] proposed a novel cost sensitive semi supervised discriminate analysis called PCSDA. Using a simple L2 approach to predict the label of unlabeled data and then learns the projection direction by incorporating costs into both labeled data and unlabeled data comparing wits CS3DA, PCCDA approaches. Using L2 approach to predict the label of unlabeled face images is more accurate and robust than the spares representation. Using CS3DA method approximates the pair wise Bayesian risk only when the classes are balanced and without outliers in data base sets. Hailing Zhou et al., [2] proposed recent advances on face recognition. 3-D data includes facial geometry information, increasing robustness to viewpoints and elimination variation compared with usual images. 3-D face recognition can achieve better recognition accuracy then the usual face recognition, although 3-D data are insensitive to illumination variations, it is still difficult to recognize faces in the options of visible light. Multimodal modalities can achieve better performance than a single modality. IR images are acquired for 2-D imaging sensors, IR face recognition also suffer from sensibility to pose variations.

Jiwen Lu et al., [3] proposed multiple statistic features and localized multi-feature metric learning method for a new image set based face recognition identification. Two kernel based metric learning algorithms called localized multi-kernel metric learning and localized multi-kernel multi-metric learning are used for extracting effectively combined multiple statistic features from face image set. Efficient kernel approximation methods are used to improve the kernel estimation speed for specific combined statistic features.

Jian yang et al., [4] proposed sparse representation classifiers steered discriminative projection with application to face recognition. Sparse representation based classifier (SRC) was used to direct the design of a dimensionality reduction method SRC - DP, in which the SRC achieved better performance and become more efficient. Meng yang et al., [5] proposed robust Kernel representation model with statistical local feature is used for face identification for different conditions, including variations of illumination, expression misalignment and pose.

\section{PROPOSED MODEL}

In this section, the new concepts of convolution of original face image with DWT of original face image along with compression of database feature vectors are introduced. The block diagram of proposed model is shown in Fig. 1.

\section{A. Face Database}

The standard face databases such as ORL, JAFFE, Yale and Indian male and Indian female [6-9]are considered to test the proposed model.

\section{B. 2D-Discrete Wavelet Transform}

The transformation is used in an image processing to remove noise effectively and also compress an image[10]:. The filter bank combinations of low pass and high pass filters are used on rows and columns of an image to derive one approximation band and three detailed bands. The approximation band is an output of low pass filter and has significant information of an image. The detailed bands are outputs of high pass and combinations of high pass-low pass filters and have detailed information such as diagonal, horizontal and vertical information of an image.

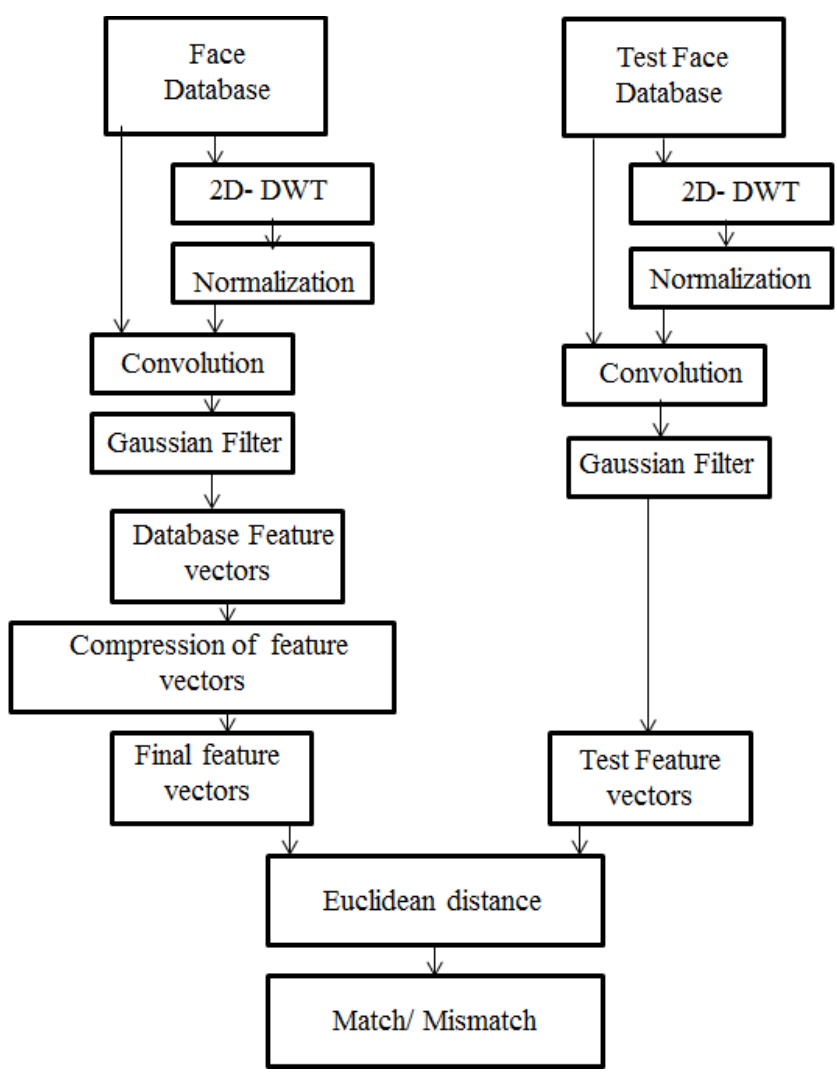

Figure1. Efficient proposed Face Recognition using Convolution and vector compression.

The DWT decomposes an image into four sub bands such as approximation band, vertical band, horizontal band and diagonal band in each level. The maximum number of decomposition levels equal to for $\mathrm{N} \times \mathrm{N}$ image size. The rows of images are passing through low pass and high pass filter to generate corresponding low and high frequency coefficients. The columns of an image are passing through low pass and high pass filter along with rows of low pass filter to generate approximation and vertical bands. The columns again passed through low pass and high pass filter along with output of row high pass filter to generate horizontal and diagonal bands. The one level DWT decomposition on an image using filter are shown in Fig. 2. 


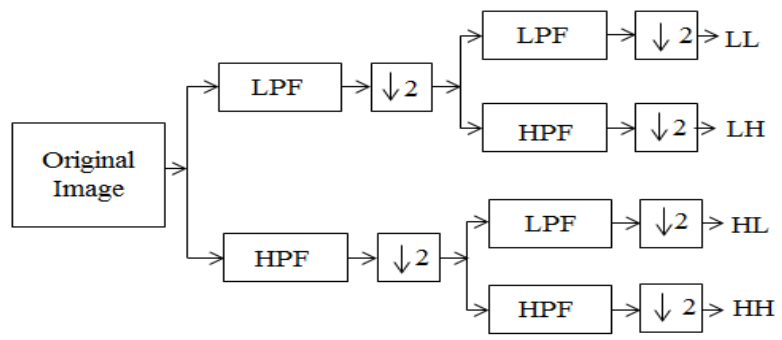

Figure 2: The one level decomposition of DWT using filter

The LL band has significant information of an original image. The LH, HL and HH bands has vertical, horizontal and diagonal information of an original image. The original image can be reconstructed by considering only LL band image and omitting other insignificant information from other bands. The DWT is applied on face databases such as JAFEE, ORL, and Yale, Indian male and Indian female of resized dimensions of $256 \times 256$. The LL band is considered for further processing as it has significant information of face images and has less noise component.

The LL band has significant information of an original image. The LH, HL and HH bands has vertical, horizontal and diagonal information of an original image. The original image can be reconstructed by considering only LL band image and omitting other insignificant information from other bands. The DWT is applied on face databases such as JAFEE, ORL, and Yale, Indian male and Indian female of resized dimensions of $256 \times 256$. The LL band is considered for further processing as it has significant information of face images and has less noise component.

\section{Normalization:}

Normalization used in the proposed method is to convert high values of LL band coefficients into a range of moderate values. The normalization is applied on LL sub-band coefficients of an image to convert high coefficients values into lower values. Each LL coefficient values are divided by maximum coefficient value to convert LL coefficient values range from 0 to 1 , as given in equation (1).

New LL coefficient values $=\frac{\text { old coefficient values }}{\text { maximum value of } L \text { L coefficient }}$

The advantage of normalization is the number of bits required to represent each LL coefficient reduces from more than eight bits to less than eight bits. The real time system complexity and memory reduces, whereas speed of computation increases.

\section{2D Convolution:}

The original face image is convolved with LL sub-band of original face image. The 2D-Convolution is used for smoothing, sharpening and edge detection of an image effectively. In the proposed method, the DWT is applied on the original face image of size $256 \times 256$ to produce four subbands, say LL, LH, HL and HH. The approximation LL Subband of size $128 \times 128$ is convolved with original spatial domain face image of size $256 \times 256$ to obtain modified original face object. The coefficients of the modified objects classify similar and dissimilar images effectively. The 2DConvolultion of two images $\mathrm{x}(\mathrm{m}, \mathrm{n})$ and $\mathrm{h}(\mathrm{x}, \mathrm{y})$ is given in equation(2).

$\mathrm{x}(\mathrm{m}, \mathrm{n}) * \mathrm{~h}(\mathrm{~m}, \mathrm{n})=\sum_{i=0}^{w-1} \quad \sum_{j=0}^{H-1} x(i, j) h(m-i, n-j)$

Where $\mathrm{w}$ and $\mathrm{H}$ are the width and height of an image. resultant output of convolution matrix of size $(\mathrm{w}+\mathrm{H}-1) *$ $(\mathrm{w}+\mathrm{H}-1)$.The example of 2D-convolution is explained by considering original matrix of size $4 * 4$ and LL band of original matrix of size $2 * 2$ is as follows:

$$
\text { Original matrix }=\left[\begin{array}{cccc}
126 & 98 & 94 & 100 \\
82 & 77 & 96 & 98 \\
80 & 104 & 146 & 129 \\
79 & 78 & 118 & 134
\end{array}\right]
$$

LL band of original Matrix $=\left[\begin{array}{cc}191.5000 & 214.000 \\ 170.5000 & 263.5000\end{array}\right]$

(4)

Normalized matrix $=\left[\begin{array}{cc}0.7268 & 0.8121 \\ 0.6471 & 1\end{array}\right]$

Original matrix $4 * 4$ is convolved with $2 * 2$ Normalized matrix to get $5 \times 5$ output convolution matrix given in equation( 6 ).

Convolution output matrix $=$

$\left[\begin{array}{ccccc}91.57 & 173.55 & 147.90 & 118.08 & 113.70 \\ 141.12 & 311.96 & 291.12 & 333.77 & 219.59 \\ 111.19 & 272.37 & 329.68 & 371.73 & 202.76 \\ 109.17 & 268.14 & 347.57 & 422.68 & 237.82 \\ 51.11 & 129.47 & 154.35 & 204.70 & 134\end{array}\right]$

The linear convolution matrix has unique coefficient values compared to original matrix coefficient values; hence the concept of convolution is used for better classification of images.

\section{E Gaussian Filter}

It is applied on output of convolution block to enhance further the quality of input face images by removing high frequency edges and helps in improving the matching accuracy. An example of Gaussian filter on convolution output matrix is as follows. The Gaussian filter mask matrix is applied on convolution output matrix is given in matrices 7 .

Gaussian filter mask $=\left[\begin{array}{ccc}0.0626 & 0.1250 & 0.0626 \\ 0.1250 & 0.2497 & 0.1250 \\ 0.0626 & 0.1250 & 0.0626\end{array}\right]$

The procedure for Gaussian filter output is as follows. 
(i). The Gaussian filter mask $(\mathrm{h}(\mathrm{x}, \mathrm{y}))$ is generated using Gaussian mask coordinators and standard deviation as given in matrix 8 and equation 11 .

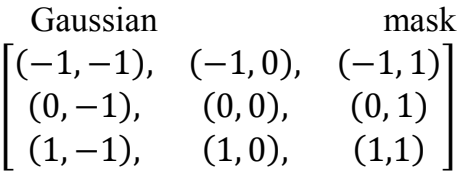

$$
\begin{aligned}
& h_{g}=e^{\frac{-\left(x^{2}+y^{2}\right)}{2 \sigma^{2}}}
\end{aligned}
$$

coordinators

Where $\sigma_{=}$standard deviation $=0.85$

$$
\begin{aligned}
& \mathrm{h}_{\mathrm{g}}(-1,-1)=e^{\frac{\left.-(-1)^{2}+(-1)^{2}\right)}{2(0.85)^{2}}}=0.25055344 \\
& \mathrm{~h}_{\mathrm{g}}(0,-1)=e^{\frac{-(0)^{2}+(-1)^{2}}{2(0.85)^{2}}}=0.500553134
\end{aligned}
$$

Similarly other coordinate coefficient values are computed and are given in matrix 11.

$$
h_{g}(x, y)=\left[\begin{array}{ccc}
0.25055344 & 0.50055314 & 0.25055344 \\
0.50055314 & 1 & 0.50055314 \\
0.25055344 & 0.50055314 & 0.0626
\end{array}\right]
$$

Gaussian filter mask coefficient are computed using equation 9 and in matrix 10.

Filter mask $=\mathrm{h}(\mathrm{x}, \mathrm{y})=\frac{h_{g}(x, y)}{\sum_{x} \Sigma_{y} h_{g}}(11)$

Where $\sum_{x} \sum_{y} h_{g}=4.004426296$

$$
h(x, y)=\left[\begin{array}{lll}
0.0626 & 0.1250 & 0.0626 \\
0.1250 & 0.2497 & 0.1250 \\
0.0626 & 0.1250 & 0.0626
\end{array}\right]
$$

(ii).Append Zeros to the surroundings of convolution output matrix.

$$
\mathrm{X}=\left[\begin{array}{ccccccc}
0 & 0 & 0 & 0 & 0 & 0 & 0 \\
0 & 91.57 & 173.55 & 147.90 & 118.08 & 113.70 & 0 \\
0 & 141.12 & 311.96 & 291.12 & 333.77 & 219.59 & 0 \\
0 & 111.19 & 272.37 & 329.68 & 371.73 & 202.76 & 0 \\
0 & 109.17 & 268.14 & 347.57 & 422.68 & 237.82 & 0 \\
0 & 51.11 & 129.47 & 154.35 & 204.70 & 134 & 0 \\
0 & 0 & 0 & 0 & 0 & 0 & 0
\end{array}\right]-
$$

(iii).Place Gaussian filter mask of size $3 * 3$ generated in step 1 on top left portion of $\mathrm{X}$ and

Multiply element by element.

$$
\begin{gathered}
\mathrm{X}_{1}=\left[\begin{array}{ccc}
0 & 0 & 0 \\
0 & 91.57 & 173.55 \\
0 & 141.12 & 311.96
\end{array}\right] *\left[\begin{array}{ccc}
0.0626 & 0.1250 & 0.0626 \\
0.1250 & 0.2497 & 0.1250 \\
0.0626 & 0.1250 & 0.0626
\end{array}\right] \\
\mathrm{X}_{1}=\left[\begin{array}{ccc}
0 & 0 & 0 \\
0 & 22.86 & 21.69 \\
0 & 17.64 & 19.52
\end{array}\right](14)
\end{gathered}
$$

$$
\sum X_{1}=81.721
$$

The $3 \times 3$ Gaussian mask is shifted right and down on $\mathrm{X}$ to compute other coefficient value of Gaussian filter output given in matrix 16.

The output of Gaussian filter $=$

$\left[\begin{array}{ccccc}81.7215 & 139.3161 & 157.6848 & 150.8504 & 98.9874 \\ 127.4855 & 230.2479 & 275.4217 & 265.6029 & 170.5192 \\ 129.4008 & 251.2676 & 326.3108 & 322.5293 & 201.6111 \\ 106.2149 & 214.7281 & 294.8670 & 302.1430 & 190.3905 \\ 59.3737 & 120.1119 & 166.9891 & 176.6282 & 115.2269\end{array}\right]$

The 2-D matrix is converted into single column vector.

F Compression of feature vectors:

The more number of sample images of single person are converted into single sample per person using compression the six column vector features of six face images of single person, say $1 \_1,2 \_1,3 \_1,4 \_1,5$ 1and $6 \_1$, are converted into single feature column vector by taking average of six column features. The advantages of converting six columns into one column are (i) Time to compare test image with database images reduces (ii) Memory requirement in real time system reduces (iii) The features of single column are more effective compared to six column vector features .

\section{G Test Image Features:}

The features are extracted from face image similar to that of database face images to form single column feature vector.

\section{H Euclidean distance:}

The distance between database features $\left(\mathrm{p}_{\mathrm{i}}\right)$ and test features $\left(\mathrm{q}_{\mathrm{i}}\right)$ is given in equation (17).

$$
E D=\sqrt{\sum_{i=1}^{N}\left(p_{i}-q_{i}\right)^{2}}
$$

Where, $\mathrm{N}=$ No of coefficients in a vector.

$\mathrm{Pi}=$ Coefficient values of vectors in the database

$\mathrm{q} i=$ Coefficient values of vectors in the test image

The ED is used to find similarities and dissimilarities among face images to test performance of biometric system. 
Table 1: Performance comparison of proposed method for different techniques with various face databases.

\begin{tabular}{|c|c|c|c|c|c|c|c|c|c|c|c|c|}
\hline \multirow[b]{2}{*}{$\begin{array}{l}\text { Databa } \\
\text { se }\end{array}$} & \multicolumn{3}{|c|}{ DWT Technique } & \multicolumn{3}{|c|}{ FFT Technique } & \multicolumn{3}{|c|}{ Convolution Technique } & \multicolumn{3}{|c|}{ Proposed Method } \\
\hline & EER & $\begin{array}{l}\% \mathrm{Opt} \\
\mathrm{TSR}\end{array}$ & $\begin{array}{l}\text { \%Max } \\
\text { TSR }\end{array}$ & EER & $\begin{array}{l}\% \mathrm{Opt} \\
\mathrm{TSR}\end{array}$ & $\begin{array}{l}\text { \%Max } \\
\text { TSR }\end{array}$ & EER & $\begin{array}{l}\% O p t \\
\text { TSR }\end{array}$ & $\begin{array}{l}\text { \%Max } \\
\text { TSR }\end{array}$ & EER & $\begin{array}{l}\% \mathrm{Opt} \\
\mathrm{TSR}\end{array}$ & $\begin{array}{l}\text { \%Ma } \\
\mathrm{x} \\
\mathrm{TSR}\end{array}$ \\
\hline JAFEE & 51 & 75 & 100 & 22 & 76 & 100 & 11 & 90 & 100 & 10 & 95 & 100 \\
\hline ORL & 30 & 62 & 90 & 36 & 60 & 83.33 & 28 & 70 & 93.33 & 18 & 73 & 93.33 \\
\hline YALE & 50 & 50 & 70 & 48 & 50 & 50 & 50 & 50 & 80 & 38 & 51 & 80 \\
\hline $\begin{array}{l}\text { Indian } \\
\text { Male }\end{array}$ & 57 & 45 & 73.33 & 55 & 45 & 93.33 & 51 & 50 & 83.33 & 40 & 55 & 83.33 \\
\hline $\begin{array}{l}\text { Indian } \\
\text { Female }\end{array}$ & 28 & 60 & 93.33 & 5 & 95 & 100 & 0 & 93.33 & 93.33 & 0 & 93.33 & 93.33 \\
\hline
\end{tabular}

\section{PERFORMANCE ANALYSIS}

In this section, the definitions of performance parameters and experimental results of various techniques are discussed.

\section{A. Definition of Performance Parameters:}

i). False Rejection Rate (FRR): It is defined as the probability of genuine person being rejected as an imposter

$$
\mathrm{FRR}=\frac{\text { numberofgenuinepersonrejected }}{\text { totalnumberofpersonsinsidethedatabase }}
$$

ii).False Acceptance Rate (FAR): Is defined as the probability of imposter being accepted as genuine persons. It is ratio of imposters accepted as genuine persons from outside the database to the total number of persons in the outside database and is computed in equation (19).

$$
F A R=\frac{\text { numberofimposteracceptedasgenuineperson }}{\text { totalnumberofpersonsintheoutsidedatabase }}
$$

iii). Equal Error Rate (ERR):Is define as error tradeoff between FRR and FAR i.e., FAR = FRR for a particular threshold value. A lower EER value indicates better performance of biometric systems.

iv. Total Success Rate (TSR):Is the measure of accuracy of biometric systems. It is the ratio of the total number of genuine persons identified correctly in the data base to the total number of persons inside the database and is computed in equation (20).

\section{TSR}

genuinepersonsidentifiedcorrectly

$\overline{\text { totalnumberofpersonsinsidethedatabase }}$

v. Optimum Total Success Rate (Opt.TSR): The value of TSR corresponding to the EER for specified threshold. vi. Maximum Total Success Rate (Max. TSR): The value of maximum TSR for specified threshold value irrespective of error values.

\section{B. Comparison of Different Techniques:}

The performance parameters viz., EER optimum TSR and max TSR are compared using different techniques for various face databases and the experimental results are tabulated in Table 1. The experimental results vary with various techniques and face databases. The maximum percentage TSR is obtained with JAFEE face database for all four techniques such as DWT, FFT, convolution and proposed techniques, since the variations in the face images are minimum. The values of EER are low with Indian female database for all four techniques. The percentage optimum TSR value varies with face databases, various techniques, maximum TSR and EER values. The percentage values of EER lower in the case of proposed technique compared to individual DWT, FFT, and Convolution techniques. The percentage values of optimum TSR and maximum TSR are higher in the case of proposed technique compared to existing techniques. . It is observed that, the performance parameter is better in the case of proposed technique compared to existing techniques. The experimental results are better in the proposed method since (i) the DWT is applied on face images and only low frequency sub band is considered to omit high frequency noise in an images. (ii) The LL sub band is convolved with original face image samples to get better features to differentiate face image samples efficiently. (iii) The feature vectors of many samples of single person are converted into single column vector with unique features to identify person efficiently. 


\section{Comparison of Proposed Method with existing Methods:}

The performance parameters such as FRR, FAR, TSR, EER and Max. TSR are computed and compared for different face databases and techniques to verify the efficiency of biometric system.

The percentage TSR of proposed method for ORL database is compared with existing algorithms presented by SwarupKumar Dandpatand SukadevMeher [11], Pallavi D. Wadakar and MeghaWankhade[12], and Murugan et al.,[13] and Ajay et al., [14].is given in Table 2. It is observed that the percentage TSR is high in the case of proposed method compared to existing algorithms. The performance of proposed method is better compared to existing algorithms for the following reasons. (i) Normalization performed on 2D DWT reduces the magnitude values of coefficients. ii). unique features are obtained using convolution. iii). Removal of high frequency noise components which help in matching accuracy using Gaussian filter .iv).Compression of Database features storing in to single column vector improves the matching speed.

Table 2: Comparison of TSR with proposed and existing algorithms

\begin{tabular}{|l|c|c|l|}
\hline $\begin{array}{c}\text { Sr. } \\
\text { No }\end{array}$ & Authors & Techniques & \%TSR \\
\hline 1 & $\begin{array}{c}\text { Swarup Kumar } \\
\text { Dandpat and } \\
\text { SukadevMeher [11] }\end{array}$ & PCA+2DPCA & 90.5 \\
\hline \multirow{2}{*}{2} & $\begin{array}{c}\text { Pallavi D. } \\
\text { Wadakar } \\
\text { and }\end{array}$ & DWT & 90 \\
\hline 3 & $\begin{array}{c}\text { MeghaWankhade[12] } \\
\text { al.,[13] }\end{array}$ & $\begin{array}{c}\text { Gabor filter + } \\
\text { DWT + PCA }\end{array}$ & 92 \\
\hline 4 & $\begin{array}{c}\text { Ajay and pankaj } \\
{[14]}\end{array}$ & N-PCA & 92.50 \\
\cline { 3 - 4 } & Proposed Method & Convolution+DWT & 93.33 \\
\hline 5 & & PCA & 90.00 \\
\hline
\end{tabular}

\section{CONCLUSION}

The face recognition matching speed is an important factor while dealing with large set of database samples. In this paper a novel technique of reducing or scaling down the feature vectors size to improve the matching speed is proposed. The face database samples and test database samples of person are subjected to DWT which generates LL band features and normalized to obtain low magnitude value. The normalized matrix is convolved with original face image pixel matrix to obtain unique matrix and passed through Gaussian filter to eliminate high frequency components. The number of feature vectors generated for different samples of single persons is compared to obtain single vector for each person, which reduces number of vectors and increases speed in real time. In future the techniques can be tested using high speed real time reconfigurable processor.

\section{References}

[1] Jianwuwan, Ming Yang, Yang Gao and Yinjuan Chen, "Pairwise Costs in Semi supervised Discriminant Analysis for Face Recognition", IEEE Transactions on Information Forensics and Security, vol.9, No.10, pp 1569-1580, October, 2014.

[2] J Hailing Zhou, AjmalMian, Lei Wei, Doug Creighton, Mo Hossny and SaeidNahavandi, "Recent Advances on Singlemodal and Multimodal Face Recognition", IEEE Transaction on Human Machine Systems, vol.44, No.6, pp 701-716, December, 2014.

[3] Jiven Lu, Gang Wann and Pierre Maulin, "Localized Multi-Feature Metric Learning for Images Set Based Face Recognition" IEEE Transaction on Circuits and Systems for Video Technology,Issue 99,pp. $1-13,2015$.

[4] JainYang, Delin Chu, Lei Zhang, Yong Xu and Jingyu Yang, "Sparse Representation Classifier Steered Discriminative Projection with Applications to Face Recognition", IEEE Transaction on Neural Networks and Learning Systems, Vol.24, No.7, pp.1023-1035July, 2013..

[5] Mang Yang, Lei Zhang, Simon Chi-Keung Shiu and David Zhang, "Robust Kernel Representation with Statistical Local Features for Face Recognition", IEEE Transaction on Neural Networks and Learning Systems, Vol.24, No.6, pp. 900-912,July, 2013.

[6] http://www.kasrl.org/jaffe download.html

[7]. http://www.camrol.co.uk

[8]. http://vision.ucsd.edu/content/yale-face-database

[9]. http://viswww.cs.umass.edu/ vidit/IndianFaceDatabase

[10 ]Arvind Bisht and Monika Gupta, "Chip Design of DWT for Image Compression", International Research Journal of Engineering and Technology, vol.2, No.4, pp 320 - 324, 2015.

[11] Swarup Kumar Dandapat and SukadevMeher, "Performance Improvement for Face Recognition using PCA and Two-Dimensional PCA," IEEE International Conference on Computer Communication and Informatics, pp. 1-5, 2013.

[12]. PallaviD.Wadkar and MeghaWankhade, "Face Recognition using Discrete Wavelet Transforms," International Journal of AdvancedEngineering Technology, vol. 3,pp. 239-242, 2012.

[13]. D Murugan, S Arumugam, K Rajalakshmi and Manish T, "Performance Evaluation of Face Recognition using Gabor Filter, Log Gabor filter and Discrete Wavelet Transform," International Journal of computer science and Information Technology, vol. 2, no. 1, pp. 125-132, 2010.

[14] Ajay Kumar Bansal and PankajChawla, "Performance Evaluation of Face Recognition using PCA and N-PCA", International Journal of Computer Applications, Vol. 76, No.8, pp 14-20, August, 2013 